Received: 30.04 .2021

Revised: 21.06 .2021

Accepted: 25.06 .2021

DOI: $10.17804 / 2410-9908.2021 .3 .045-054$

\title{
ON THE RATIONAL CHOICE OF THE PROPERTIES OF STRUCTURAL ELEMENTS CONSTITUTING UNIDIRECTIONAL COMPOSITIONS
}

\author{
V. V. Struzhanov \\ Institute of Engineering Science, Ural Branch of the Russian Academy of Sciences, \\ 34 Komsomolskaya St., 620049, Ekaterinburg, Russian Federation \\ iD http://orcid.org/0000-0002-3669-2032 ه stru@imach.uran.ru \\ Corresponding author: E-mail: stru@imach.uran.ru \\ Address for correspondence: 34 Komsomolskaya St., 620049, Ekaterinburg, Russian Federation \\ Tel.:+7(343)362 3019
}

A technique is proposed that makes it possible to implement a rational choice of fibers constituting a unidirectional tensile composite. The choice is made in such a way as to avoid catastrophic failure. The tensile properties of the fibers are characterized by complete deformation diagrams with falling branches. It is shown that the fibers must have different properties since it is impossible to avoid a catastrophe (dynamic fracture of the composite) if the properties are identical.

Keywords: unidirectional composite, tension, rational choice, complete deformation diagram, fracture.

\section{Acknowledgment}

The work was performed according to the state assignment on theme No. AAAA-A18118020790145-0.

\section{References}

1. Bataev A.A., Bataev V.A. Kompozitsionnye materialy: stroenie, poluchenie, primenenie [Composite Materials : Structure, Production, application: Textbook]. Novosibirsk, Izd-vo NGTU Publ., 2002, 384 p. (In Russian).

2. Mikhailin Yu.A. Konstruktsionnye polimernye kompozitsionnye materialy [Structural Polymer Composite Materials]. SPb, Nauchnye Osnovy i Tekhnologii Publ., 2010, 822 p. (In Russian).

3. Matthews F., Rawlings R. Kompozitsionnye materialy i konstruktsii, Engl. transl. [Composite materials. Engineering and science, Cambridge, CRC Press, Woodhead Publishing, 1999, 480 p.]. Moscow, Technosphera Publ., 2004, 407p. (In Russian).

4. Warden K. Novye intellektualnue materialy i konstrukts [New Intellectual Materials and Designs]. Moscow, Technosphere Publ., 2006, 223 p. (In Russian).

5. Pobedrya B.Ye. Mechanics of composite materials [Mekhanika kompozitsionnykh materialov]. MGU Publ., 1984, 336 p. (In Russian).

6. Garishin, O.K. and Lebedev, S.N., Investigation of structure stresses in dispersion-filled elastomeric nanocomposite, Mekh. Kompoz. Mater. Konstr., 2006, vol. 12, No. 3, pp. 289.

7. Zhigun V.I., Plume E.Z. Evaluation of carrying capacity of carbon-carbon composites in plane stress state. Mekhanika kompozitsionnykh materialov i konstruktsiy, 2017, vol. 23, No. 1, pp. 25-40.

8. Khaliulin V.I., Shapaev I.I. Technology of Composite Parts Production. Kazan, KGTU Publ., 2003, 368p. (In Russian). 
9. Perepelkin K.E. Armiruyushchie volokna i voloknistye polimernye kompozity [Reinforcing fibers and fibrous polymer composites]. Moscow, NOT Publ., 2009, 658 p. (In Russian).

10. Sedov L.I. Mekhanika sploshnoy sredy [Continuum mechanics, vol. 1]. M, Nauka Publ., 1970, 492p.

11. Poston T. and Stewart I. Teoriya katastrof $i$ ee prilozheniya [Catastrophe Theory and Its Application, London, Pitman, 1978]. Moscow, Mir Publ., 1980. (In Russian).

12. Vildeman V.E., Chausov N.G. Conditions of strain softening upon stretching of the specimen of special configuration. Zavodskaya Laboratoriya. Diagnostika Materialov, 2007, vol. 73, No. 10, pp. 55-59. (In Russian).

13. Wildemann V.E., Tretyakov M.P. Tests of Materials with Construction of Complete Deformations Curves. Probl. Mashinostr. Nadezhn. Mashin, 2013, No. 2, pp. 93-98. (In Russian).

14. Struzhanov V.V., Korkin A.V., Chaikin A.E. One approach to determination of the ultimate load-bearing capacity of mechanical systems with softening elements. Vestn. Samar. Gos. Tekhn. Univ., Ser. Fiz.-Mat. Nauki [J. Samara State Tech. Univ., Ser. Phys. Math. Sci.]. 2018, vol. 22, No. 4, pp. 762-773. DOI: 10.14498/vsgtu1624. (In Russian).

15. Struzhanov V.V., Mironov V.I. Deformatsionnoe razuprochnenie materiala $v$ elementakh konstruktsiy [Deformational softening of material in structural elements]. Yekaterinburg, Ural Branch of the Russian Academy of Sciences Publ., 1995, 190 p. (In Russian).

16. Struzhanov V.V., Korkin A.V. Regarding stretching process stability of one bar system with softening elements. Vestnik Uralskogo Gosudarstvennogo Universiteta Putey Soobshcheniya, 2016, 3 (31), pp. 4-17. DOI: 10.20291/2079-0392-2016-3-4-17. (In Russian).

17. Pars L. Analytical Dynamics, Engl. transl., Moscow, Mir Publ., 1989, 655 p.

18. Horn R., Johnson C. Matrix analysis, Engl. transl., Moscow, Mir Publ., 1989, 655 p. 
Подана в журнал: 30.04.2021

УДК 539.3:539.382

DOI: $10.17804 / 2410-9908.2021 .3 .045-054$

\title{
О РАЦИОНАЛЬНОМ ВЫБОРЕ СВОЙСТВ СТРУКТУРНЫХ ЭЛЕМЕНТОВ, СОСТАВЛЯЮЩИХ ОДНОНАПРАВЛЕННЫЕ КОМПОЗИЦИИ
}

\author{
В. В. Стружанов
}

\author{
Федеральное государственное бюджетное учреждение науки \\ «Институт машиноведения Уральского отделения Российской академии наук», \\ д. 34 ул. Комсомольская, 620049, г. Екатеринбург, Российская Федерация \\ iD http://orcid.org/0000-0002-3669-2032 ه stru@imach.uran.ru
}

\author{
Ответственный автор. Электронная почта: stru@imach.uran.ru \\ Адрес для переписки: ул. Комсомольская, 34, г. Екатеринбург, Российская Федерация \\ Тел.:+7(343)362-30-19
}

\begin{abstract}
Предложена методика, позволяющая реализовать рациональный выбор волокон, составляющих однонаправленный композит, работающий на растяжение. Выбор осуществляется таким образом, чтобы избежать катастрофического разрушения. Свойства волокон при растяжении характеризуются полными диаграммами деформирования с падающими ветвями. Показано, что волокна должны иметь разные свойства, так как при их совпадающих свойствах катастрофы (динамического разрушения композита) не возможно избежать.
\end{abstract}

Ключевые слова: однонаправленный композит, растяжение, рациональный выбор, полная диаграмма деформирования, разрушение.

\section{1. Введение}

Композиционные материалы благодаря их уникальным свойствам находят широкое применение в промышленности при изготовлении различных элементов конструкций [1-4].

Основное внимание при разработке материала для конкретного изделия уделяется расчету напряженно-деформированного состояния в компонентах композита с целью оценить их прочность и сопротивление внешним нагрузкам [5-7].

Как правило рассматриваются различные варианты компоновки элементов композита, из которых выбирают наиболее подходящие [8]. Вопросом же оптимального проектирования материала по заданным внешним нагрузкам на изделие уделяется значительно меньше внимания.

Одним из распространенных классов композиционных материалов являются однонаправленные композиты, обладающие повышенной прочностью при растяжении вдоль волокон [9]. В работе разработана методика рационального выбора волокон, чтобы избежать динамического разрушения. Свойства волокон при растяжении описываются полными диаграммами деформирования с падающими ветвями. Под разрушением понимается невозможность равновесия [10].

Для анализа устойчивости равновесия применяется аппарат математической теории катастроф [11]. Установлено, что при совпадающих свойствах волокон динамическое разрушение композита неизбежно.

\section{2. Постановка задачи}

Рассмотрим композицию, составленную из однонаправленных элементов (однонаправленный композит). Нагружение осуществляем таким образом, чтобы все элементы работали только на растяжение. При этом элементы могут находиться как в стадии упрочнения,

Struzhanov V. V. On the rational choice of the properties of structural elements constituting unidirectional compositions // Diagnostics, Resource and Mechanics of materials and structures. - 2021. - Iss. 3. - P. 45-54. - DOI: 10.17804/2410- 
так и в стадии разупрочнения. Полагаем также, что прочность связующего, объединяющего элемента в единое целое пренебрежимо мало. Тогда данную композицию можно представить в виде системы из $\mathrm{n}$ параллельных стержней, подвергаемой растяжению в специальном устройстве (рис. 1).

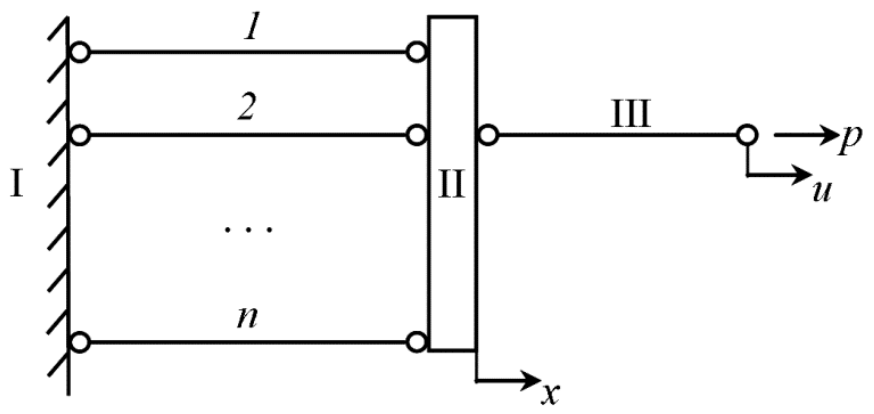

Рис. 1. Стержневая система, представляющая аналог однонаправленной композиции: I - неподвижная жесткая стенка; II - поршень; III - стержень

Одним концом стержни соединены с неподвижной жесткой стенкой I, а другим с абсолютно жестким поршнем II, который перемещается на расстояние $x$ под действием растягивающего усилия, передающегося стержнем III. Стержень III упругий и имеет жесткость при растяжении, равную $C$. Растяжение системы осуществляем, задавая перемещение $u$ свободному концу стержня III (жесткое нагружение) или прикладывая растягивающую силу $p$ (мягкое нагружение). Нагружение происходит квазистатически при постоянной температуре.

Деформационные свойства растягиваемых стержней $1,2, \ldots, n$ определяют, соответственно, функции $q_{1}(x), q_{2}(x), \ldots q_{n}(x)$, где $x$ - удлинение стержней. Эти функции представляют собой полные диаграммы растяжения и имеют восходящие и ниспадающие до нуля ветви. Восходящие ветви характеризуют свойства стержней на стадиях упругости и упрочнения, ниспадающие ветви - на стадии разупрочнения (неустойчивого растяжения) $[12,13]$.

При упругом деформировании жесткость стержней равна $\lambda_{1}, \lambda_{2}, \ldots, \lambda_{n}$, свойства неупругого деформирования заданы касательными модулями к функциям $q_{n}(x)$, а именно, $\lambda_{1}^{p}, \lambda_{2}^{p}, \ldots, \lambda_{n}^{p}$. Разгрузка происходит по линейному закону с модулями $\lambda_{1}, \lambda_{2}, \ldots, \lambda_{n}$.

При жестком нагружении система имеет одну обобщенную координату $x$ (параметр состояния) и один параметр управления $u$. При мягком нагружении система имеет две обобщенные координаты $x, u$ (параметр состояния) и один параметр управления $p$.

Так как разрушение происходит в момент потери устойчивости процесса растяжения системы $[10,14]$, то задача состоит в следующем: необходимо подобрать стержни с такими свойствами, чтобы потеря устойчивости происходила как можно позднее или вообще возможно было бы избежать потери устойчивости и провести процесс растяжения полностью равновесно.

\section{3. Система с одним разупрочняющимся стержнем}

Приведем общие положения по определению момента потери устойчивости композиции в виде системы параллельных стержней на примере растяжения одного разупрочняющегося стержня $[15,16]$.

Выпишем лагранжиан системы сначала при жестком нагружении. Имеем: 


$$
W_{1}^{u}=\int_{0}^{x} q_{1}(x) d x+0,5 C(u-x)^{2} .
$$

Далее, используя уравнение Лагранжа II рода [11], получаем уравнение равновесия

$$
\frac{\partial W_{1}^{u}}{\partial x}=q_{1}(x)-C(u-x)=0 .
$$

Решения уравнения (1) при квазистатическом возрастании параметра u есть критические точки функции $W_{1}^{u}$. Вырожденные критические точки, где происходит переход в неустойчивое состояние равновесия, определяются совместным решением уравнения (1) и уравнения [11]:

$$
\frac{\partial^{2} W_{1}^{u}}{\partial x^{2}}=\frac{\partial q_{1}}{\partial x}+C=\lambda_{1}^{p}(x)+C=0 .
$$

Следовательно, потеря устойчивости системы произойдет в том случае, если на падающей ветви диаграммы деформирования стержня касательный модуль $\lambda_{1}^{p}=-C$.

В случае мягкого нагружения лангранжиан системы равен [17]

$$
W_{1}^{p}=W_{1}^{u}-\int_{0}^{u} p d u
$$

В силу того, что в данном случае имеется две обобщенные координаты, получаем систему уравнений равновесия

$$
\frac{\partial W_{1}^{p}}{\partial x}=\frac{\partial W_{1}^{u}}{\partial x}=q_{1}(x)-C(u-x)=0 ; \quad \frac{\partial W_{1}^{p}}{\partial u}=C(u-x)-p=0 .
$$

Вырожденные критические точки системы определяются совместным решением уравнений (2) и уравнения, полученного приравниванием к нулю детерминанта матрицы Гессе лангранжиана, которая имеет вид $[11,18]$ :

$$
H\left(W_{1}^{p}\right)=\left(\begin{array}{ll}
\frac{\partial^{2} W_{1}^{p}}{\partial x^{2}} & \frac{\partial^{2} W_{1}^{p}}{\partial u \partial x} \\
\frac{\partial^{2} W_{1}^{p}}{\partial u \partial x} & \frac{\partial^{2} W_{1}^{p}}{\partial u^{2}}
\end{array}\right)=\left(\begin{array}{cc}
\lambda_{1}^{p}(x)+C & -C \\
-C & C
\end{array}\right)
$$

В результате приравнивания детерминанта к нулю получаем уравнение:

$$
\operatorname{det} H\left(W_{1}^{p}\right)=C\left(\lambda_{1}^{p}+C\right)-C^{2}=0 .
$$

Тогда потеря устойчивости системы произойдет при $\lambda_{1}^{p}(x)=0$, т. е. когда будет достигнута наивысшая точка на диаграмме деформирования разупрочняющегося стержня.

\section{4. Система с двумя разупрочняющимися стержнями}

В этом случае лагранжиан системы имеет вид: 


$$
W_{2}^{u}=\int_{0}^{x} q_{1}(x) d x+\int_{0}^{x} q_{2}(x) d x+0,5 C(u-x)^{2}
$$

Уравнение равновесия

$$
\frac{\partial W_{2}^{u}}{\partial x}=q_{1}(x)+q_{2}(x)-C(u-x)=0 .
$$

Вырожденные критические точки, в которых происходит смена типа равновесия, определяются из уравнения

$$
\frac{\partial^{2} W_{2}^{u}}{\partial x^{2}}=\frac{\partial q_{1}}{\partial x}+\frac{\partial q_{2}}{\partial x}+C=\lambda_{1}^{p}(x)+\lambda_{2}^{p}(x)+C=0 .
$$

Рассмотрим фазовую плоскость с декартовыми координатами $\lambda_{1}^{p}, \lambda_{2}^{p}$. На этой плоскости равенство (3) задает прямую $\lambda_{1}^{p}+\lambda_{2}^{p}+C=0$ (рис. 2; прямая 1), разделяющую области устойчивости системы при квазистатическом возрастании величины $u$ (зона I) и неустойчивости (зона II).

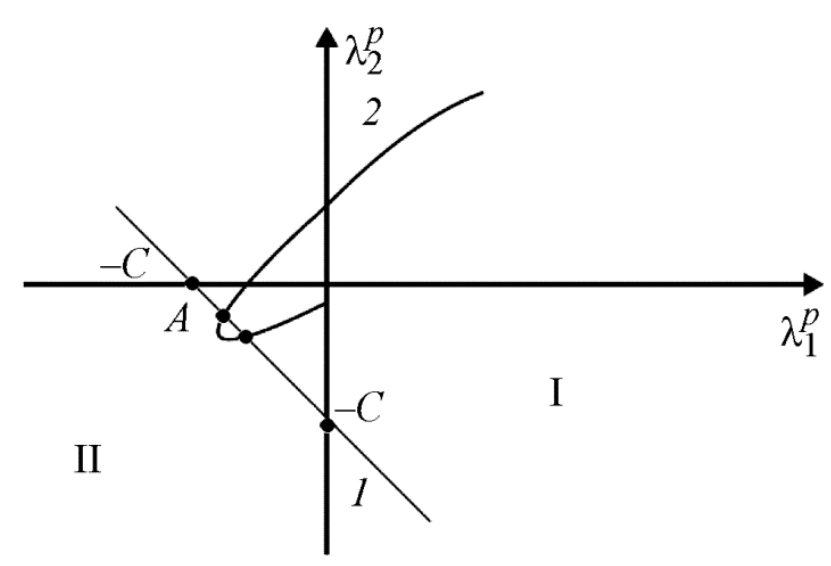

Рис. 2. Фазовый портрет процесса растяжения

Параметрическое уравнение кривой, отражающей процесс деформирования разупрочняющихся стержней, задано функциями $\lambda_{1}^{p}(x), \lambda_{2}^{p}(x)$. Изменяя параметр $x$, получаем кривую 2 (рис. 2). При пересечении кривой 2 и линии 1 в некоторой точке $A$ происходит скачкообразный переход системы в новое положение равновесия (потеря устойчивости процесса растяжения)

Очевидно, что потери устойчивости не произойдет, если кривая 2 не будет пересекать прямую 1. Следовательно, расстояние от каждой точки $\left\{\lambda_{1}^{p}(x) \lambda_{2}^{p}(x)\right\}$ до прямой 1 должно быть всегда больше или равно нулю для каждого значения $x$ :

$$
\delta=\frac{\lambda_{1}^{p}(x)+\lambda_{2}^{p}(x)+C}{\sqrt{2}} \geq 0 .
$$

Используя условие (4), можно осуществить рациональный выбор материалов разупрочняющихся стержней по следующей схеме. Пусть имеется набор стержней с характеристиками $\lambda_{k}^{p}(x)(k=1,2, \ldots, K)$. Сформируем множество функций: 


$$
\delta_{e m}=\frac{\lambda_{e}^{p}(x)+\lambda_{m}^{p}(x)+C}{\sqrt{2}},(e, m=1,2, \ldots K)
$$

Затем находим $\min _{x} \delta_{e m}$, т. е. минимальные расстояния точек кривых, определяемых функциями $\lambda_{e}^{p}(x)$ и $\lambda_{m}^{p}(x)$ от прямой 1 . После этого вычисляем $\max _{e, m} \min _{x} \delta_{e m}$. Отсюда получаем такие значения $e$ и $m$, при которых минимальное расстояние точек фазовой кривой 2 от прямой 1 максимально. Если для данных $e$ и $m$ выполняется условие (4), то растяжение системы с характеристиками $\lambda_{e}^{p} u \lambda_{m}^{p}$ происходит без катастрофического скачка и система обладает необходимой живучестью, т. е. имеет способность сохранять по крайней мере ограниченную несущую способность при повреждении или разрушении отдельных элементов. В том случае, когда в данном наборе материалов нет комбинаций, обеспечивающих выполнение условия (4), необходимо выключить в этот набор новые материалы.

Отметим, что при равенстве свойств разупрочняющихся стержней $\lambda_{1}^{p}(x)=\lambda_{2}^{p}(x)=$ $=\lambda^{p}(x)$. Пусть деформирования на фазовой плоскости представляется прямой 3 (рис. 3 ), которая пересекает прямую 1 в точке $B$ с координатами $\lambda_{1}^{p}=-\frac{C}{2} ; \lambda_{2}^{p}=-\frac{C}{2}$.

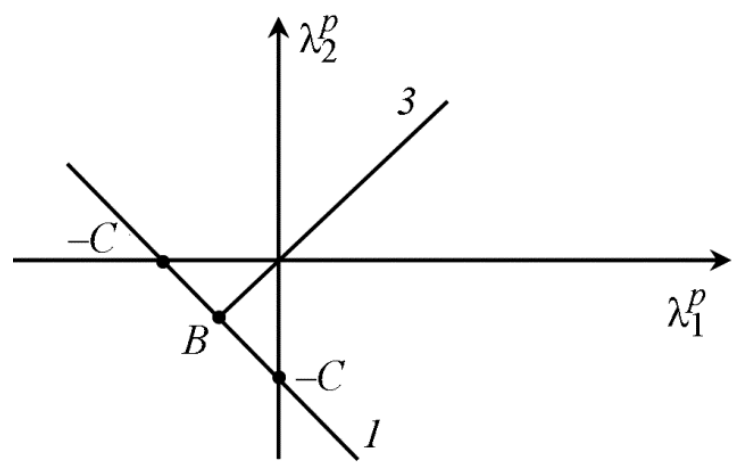

Рис. 3. Фазовый портрет растяжения стержней с одинаковыми свойствами

При увеличении параметра $u$ потеря устойчивости системы обязательно происходит и наступает раньше чем в системе с одним стержнем.

Перейдем к мягкому нагружению. Имеем Лагранжиан:

$$
\mathrm{W}_{2}^{p}=\int_{0}^{x} q_{1}(x) d x+\int_{0}^{x} q_{2}(x) d x+0,5 C(u-x)^{2}-\int_{0}^{u} p d u
$$

и уравнения равновесия

$$
\begin{gathered}
\frac{\partial W_{2}^{p}}{\partial x}=q_{1}(x)+q_{2}(x)-C(u-x)=0, \\
\frac{\partial W_{2}^{p}}{\partial u}=C(u-x)-p=0 .
\end{gathered}
$$

Вырожденные критические точки определяются уравнением, получающимся приравниванием к нулю детерминанта матрицы Гессе: 


$$
\begin{aligned}
& \left|\begin{array}{ll}
\frac{\partial^{2} W_{2}^{p}}{\partial x^{2}} & \frac{\partial^{2} W_{2}^{p}}{\partial x \partial u} \\
\frac{\partial^{2} W_{2}^{p}}{\partial x \partial u} & \frac{\partial^{2} W_{2}^{p}}{\partial x^{2}}
\end{array}\right|=\left|\begin{array}{cc}
\lambda_{1}^{p}(x)+\lambda_{2}^{p}(x)+C & -c \\
-C & C
\end{array}\right|= \\
& =\left(\lambda_{1}^{p}+\lambda_{2}^{p}+C\right) C-C^{2}=\lambda_{1}^{p}(x)+\lambda_{2}^{p}(x)=0 .
\end{aligned}
$$

На фазовой плоскости равенство (5) задает прямую

$$
\lambda_{1}^{p}+\lambda_{2}^{p}=0
$$

проходящую через начало координат под углом $\frac{\pi}{4}$ к осям координат. Эта прямая разделяет области устойчивости системы при квазистатическом возрастании величины $p$ (зона I) и неустойчивости (зона II) (рис. 2).

Потеря устойчивости процесса растяжения не произойдет, если фазовая кривая (5) не будет пересекать прямую (б). В этом случае расстояние от каждой точки фазовой кривой до прямой (6) должно быть больше или равно нулю:

$$
\gamma=\frac{\lambda_{1}^{p}(x)+\lambda_{2}^{p}(x)}{\sqrt{2}} \geq 0 .
$$

Так же осуществим рациональный выбор материалов из набора стержней с характеристиками $\lambda_{k}^{p}(x)$. Сформируем множество функций

$$
\gamma_{e m}=\frac{\lambda_{e}^{p}(x)+\lambda_{m}^{p}(x)}{\sqrt{2}}
$$

Находим $\max _{e, m} \min _{x} \gamma_{e m}$. Отсюда получаем такие значения $e$ и $m$, при которых расстояние точек фазовой кривой 5 от прямой 6 максимально.

Отметим, что при равенстве свойств разупрочняющихся стержней потеря устойчивости (разрушения) обязательно произойдет, когда $\lambda_{1}^{p}=\lambda_{2}^{p}=0$.

\section{5. Общий случай}

Пусть система имеет $n$ разупрочняющихся стержней. Тогда, опираясь на методику, изложенную выше, при жестком нагружении имеем уравнение равновесия:

$$
q_{1}(x)+\cdots+q_{n}(x)-C(u-x)=0
$$

и вырожденные критические точки лагранжиана определяются из уравнения

$$
\frac{\partial q_{1}}{\partial x}+\cdots+\frac{\partial q_{n}}{\partial x}+C=\lambda_{1}^{p}(x)+\cdots+\lambda_{2}^{p}(x)+C=0 .
$$

В фазовом $n$-мерном евклидовом пространстве с декартовой системой координат $\lambda_{1}^{p}, \ldots, \lambda_{n}^{p}$ равенство (7) задает плоскость

$$
\lambda_{1}^{p}+\cdots+\lambda_{n}^{p}+C=0,
$$

разделяющую области устойчивости системы при квазистатическом возрастании величины $u$ и неустойчивости. 
Система всегда находится в области устойчивости, если расстояние точек фазовой кривой, заданной функциями $\lambda_{1}^{p}(x), \ldots, \lambda_{n}^{p}(x)$, будет больше или равно нулю:

$$
\beta=\frac{\lambda_{1}^{p}(x)+\cdots+\lambda_{n}^{p}(x)+C}{\sqrt{n}} \geq 0 .
$$

Тогда рациональный выбор материалов разупрочняющихся стержней осуществляем по следующей схеме. Используя набор стержней с характеристиками $\lambda_{k}^{p}(x)$, сформируем множество функций:

$$
\beta=\frac{\lambda_{e}^{p}(x)+\cdots+\lambda_{m}^{p}(x)+C}{\sqrt{n}}
$$

Вычисляем $\max _{e, m} \min _{x} \beta_{e m}$ и отсюда получаем значение $e$ и $m$, при которых расстояние точек фазовой кривой от плоскости (8) будет минимально.

Отметим, что при равенстве свойств стержней $\lambda_{1}^{p}=\lambda_{2}^{p}=\cdots=\lambda_{n}^{n}=\lambda^{p}$ потеря устойчивости растяжения обязательно наступает, когда касательные модули стержней равняются $\lambda_{n}^{p}$. Этот случай практически не отличается от мягкого нагружения системы с одним разупрочняющимся стержнем, когда потеря устойчивости растяжения происходит при $\lambda^{p}=0$.

При мягком нагружении, приравнивая детерминант матрицы Гессе к нулю, получаем:

$$
\lambda_{1}^{p}(x)+\cdots+\lambda_{n}^{p}(x)=0 .
$$

Используя данное равенство, формируем множество функций:

$$
\varphi_{e m}=\frac{\lambda_{e}^{p}(x)+\cdots+\lambda_{m}^{p}(x)}{\sqrt{n}} .
$$

Тогда рациональный выбор стержней определяем из условия $\max _{e, m} \min _{x} \varphi_{e m}$.

\section{6. Заключение}

Разработана методика рационального выбора элементов однонаправленных композитов с целью увеличения живучести композиции при растяжении вдоль волокон. Установлено, что равенство свойств волокон не способствует живучести композиции, а наоборот, приводит к быстрому разрушению.

\section{Благодарность}

Работа выполнена в соответствие с государственным заданием по теме № АAАAA18-118020790145-0.

\section{Литература}

1. Батаев А. А., Батаев В. А. Композиционные материалы: строение, получение, применение. - Новосибирск : Изд-во НГТУ, 2002. - 384 с.

2. Михайлин Ю. А. Конструкционные полимерные композитные материалы. - М. : Изд-во Научные основы и технологии, 2008. - 822 с.

3. Мэттьюз Ф., Ролингс Р. Композиционные материалы и конструкции. - Механика и технологии. - М. : Техносфера, 2004. - 407 с. 
4. Уорден К. Новые интеллектуальные материалы и конструкции. - М. : Техносфера, 2006. -223 c.

5. Победря Б. Е. Механика композиционных материалов. - М. : Изд-во МГТУ, 1984. $336 \mathrm{c}$.

6. Гаришин О. К., Лебедев С. Н. Исследование структурных напряжений в дисперсно наполненных эластомерных нанокомпозитах // Механика композиционных материалов. 2006. - № 3. - С. 289-299.

7. Ж Жигун В. И., Плуме Э. З. Оценка несущей способности углерод-углеродных композитов при плоском напряженном состоянии // Механика композиционных материалов. 2017. - Т. 23, № 1. - С. 25-40.

8. Халиулин В. И., Шапаев И. И. Технология производства композитных изделий : учебное пособие. - Казань : Изд-во КГТУ, 2003. - 368 с.

9. Перепелкин К. Е. Армирующие волокна и волокнистые полимерные композиты. М. : Изд-во Научные основы и технологии, 2009. - 658 с.

10. Седов Л. И. Механика сплошной среды. Т.1. - М. : Наука, 1970. - 492 с.

11. Постон Т. Стюарт И. Теория катастроф и ее приложения. - М. : Мир, 1980. - 608 с.

12. Вильдеман В. Э., Чаусов Н. Г. Условия деформационного разупрочнения материала при растяжении образца специальной конфигурации // Заводская лаборатория: Диагностика материалов, 2007. - Т. 73, № 10. - С. 55-59.

13. Вильдеман В. Э., Третьяков М. П. Испытания материалов с построением полных диаграмм деформирования // Проблемы машиностроения и надежности машин. - 2013. - № 2. C. 93-98.

14. Стружанов В. В., Коркин А. В., Чайкин А. Е. Об одном подходе к определению предельной несущей способности механических систем с разупрочняющимися элементами // Вестн. Сам. Гос. тех. ун-та сер. Физ.-мат науки. - 2018. - Т. 22, № 4. - С. 762-773.

15. Стружанов В. В., Миронов В. И. Деформационное разупрочнение материала в элементах конструкций. - Екатеринбург : Изд-во УрО РАН, 1995. - 190 с.

16. Стружанов В. В., Коркин А. В. Об устойчивости процесса растяжения одной стержневой системы с разупрочняющимися элементами // Вестник Уральского гос. ун-та путей сообщения. - 2016. - № 3. - С. 4-17.

17. Парс Л. Аналитическая динамика / пер. с англ. - М. : Мир, 1989. - 655 с.

18. Хорн Р., Джонсон Ч. Матричный анализ / пер. с англ. - М. : Мир, 1989. - 655 с. 